\title{
Publisher's Note: Control of flow around a circular cylinder for minimizing energy dissipation [Phys. Rev. E 90, 053008 (2014)]
}

Hiroshi Naito and Koji Fukagata

(Received 17 December 2014; published 23 December 2014)

DOI: 10.1103/PhysRevE.90.069902

PACS number(s): 47.85.L-, 99.10.Fg

This paper was published online on 17 November 2014 with an incorrect figure. Figure 8(a) has been replaced as of 18 December 2014. The figure is incorrect in the printed version of the journal; therefore for the benefit of the print readership, the figure has been replicated below.
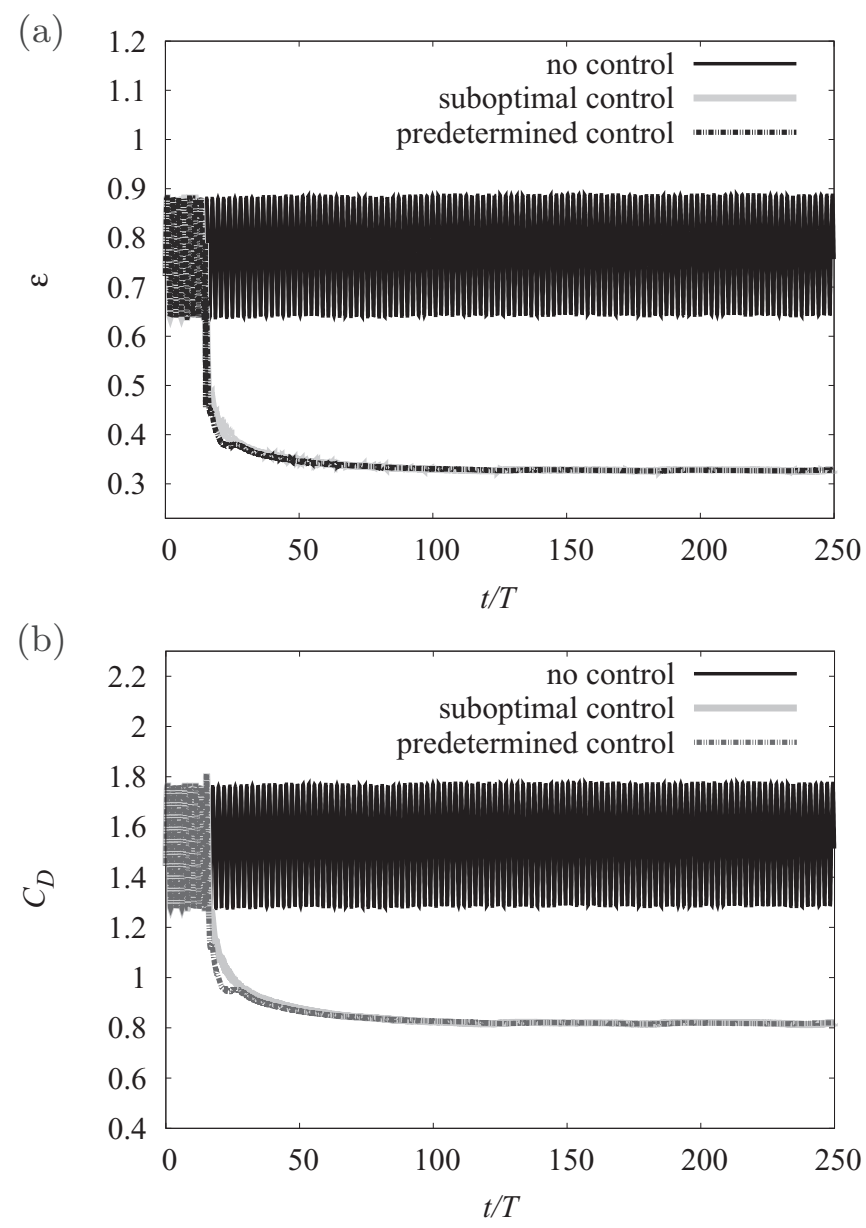

FIG. 8. Time traces in the predetermined control case $(\operatorname{Re}=1000)$ : (a) energy dissipation, $\varepsilon$; (b) drag coefficient, $C_{D} \cdot \theta^{\prime}=180^{\circ}-\theta$. The dissipation rate $\varepsilon$ is made dimensionless by $\rho, U_{\infty}$, and $D$. 